\title{
Using Operational Architecture to Model Embedded Software
}

\author{
Sang-Uk Jeon ${ }^{\dagger} \quad$ Jang-Eui Hong ${ }^{\ddagger} \quad$ Doo-Hwan Bae ${ }^{\dagger}$ \\ $\dagger$ Division of Computer Science \\ Department of EE and CS \\ KAIST, Korea \\ \{sujeon, bae\}@ se.kaist.ac.kr \\ $\ddagger$ School of Electrical\& \\ Computer Engineering \\ Chungbuk National Univ., Korea \\ jehong@chungbuk.ac.kr
}

\begin{abstract}
Almost methods in embedded software modeling take software architecture as one of the crucial parts of the design process. Since a principal role of embedded software is the interaction with its environments, architectures that describe the dynamic aspects are also needed. Although such architectural view is significantly important in embedded software development, the existing approaches do not practically guide the architectural design based on the dynamic aspects. In this paper, we propose an operational architecture to represent the dynamic aspects of an embedded software. Also we explain how to use our operational architecture in the process of embedded software modeling. Our operational architecture effectively reflects interactionbased dynamic behavior of the system, and gives traceability between requirements and design model.
\end{abstract}

\section{Introduction}

The complexity of embedded software is growing rapidly because of the demand for new and complicated features[13] such as shown in mobile phone, home-assistant robot, automobile, etc. A critical key to develop embedded software with such complexity is the ability to develop high-quality ones on time[11].

In order to support efficient development of the embedded software, many modeling methods were proposed[5, $10,15]$. In those methods, the architecture is one of the crucial parts of the design process. The software architecture is the structure or structures of the system, which comprise software elements, the externally visible properties of those elements, and the relationships among them[1]. The architectures in most modeling approaches, mainly focus on static structures of the system. However, since a principal role of embedded software is the interaction with the physical world[13], architectures that describe dynamic aspects, especially interactions with the environments, are also needed.

In this paper, we propose an operational architecture to represent the interaction-based behavior of embedded software. Operational architecture defines the activities, interactions between them, and their relationships. Those activities are often regarded also as functions. Our approach constructs operational architecture from use case scenarios(shortly scenarios). A couple of steps in a scenario is captured as an activity. And by capturing the interactions between those activities, the operational architecture is constructed.

There are three types of operational architecture in our approach. The scenario-level operational architecture reflects the dynamic behaviors which are explicitly or implicitly contained within a scenario. The use-case-level operational architecture reflects those from one use case and the system-level operational architecture reflects those of the global system view. We first formally define the operational architecture and describe each element in it. Then we show how an scenario-level operational architecture for a scenario is described. From those scenario-level operational architecture, use-case-level ones are constructed. To create the system-level operational architecture, those usecase-level operational architectures are composed. So we define two composition functions for scenario-level operational architecture and one for use-case-level operational architecture. We also suggest how to derive behavioral models of structured modeling or object-oriented modeling from our operational architecture. Our modeling approach enables seamless construction of design models from requirements because of the good applicability of use cases, thus is capable of providing models that are intuitive and easy to understand. In our approach, we use UML 2.0 notations $[2,6,7,14]$ to graphically represent the operational architecture for putting together with existing methods. 
The remainder of this paper is organized as follows. Section 2 presents the formal definition of our operational architecture and how it is constructed from various scenarios. Section 3 discusses about related works. Finally, conclusions and future work are discussed in Section 4.

\section{Definitions of Operational Architecture}

An operational architecture consists of a set of software components, a set of flows between the components, and a set of constraints on the components.

Definition 1 (Operational architecture). An operational architecture $\mathcal{A}$ is a 5-tuple $\left(\mathcal{C}, \mathcal{R}, q_{\text {init }}, q_{\text {final }}, \mathcal{S}\right)$ where

- $\mathcal{C}$ is a set of software components,

- $\mathcal{R}=\mathcal{C} \times\{O, D, M, F, J, I\} \times \mathcal{C} \times T$ is a transition relation,

- $q_{\text {init }}$ is the initial component,

- $q_{\text {final }}$ is the final component,

- $\mathcal{S} \subseteq \mathcal{C} \times \mathcal{C} \times T$ is a set of time constrains on software components.

A software component $\mathrm{c} \subseteq \mathcal{C}$ is a modularized function. It represents a task in embedded software. A software component can be classified into three types; functional component, interface component, and reference component. The functional components represent functional behavior that produce appropriate outputs from given inputs. The interface components are in charge of communicating with the environments by exchanging events. The reference components represent another operational architecture. In case of scenario-level operational architecture, there are no reference components. The concept of the reference components enables our approach to construct operational architectures with hierarchy. The set $\mathcal{S}$, represent time constraints between two software components.

A transition relation $\mathcal{R}$ represents relationships between software components. There are five kinds of relationship between two components; $\operatorname{ordinary}(\mathrm{O})$, decision(D) with guard condition $(\mathrm{T})$, merge $(\mathrm{M})$, fork $(\mathrm{F})$, join $(\mathrm{J})$ and interruptable(I). Here, we incorporate the concept of interruptable region of UML activity diagrams[14] to our operational architecture. The execution of software components $\mathcal{C}^{\prime} \subseteq \mathcal{C}$ in an interruptable region could be aborted by some interrupts. Those interrupts are generated by accepting events from the environment in some interface components in $\mathcal{C}$. If an interrupt occurs in an interface component $c_{i}$, then all executions in the region are terminated and a software component which is connected to $c_{i}$ is executed. Figure 1 shows an example. The dotted box represents the interruptable region. Assume that the system receives an external event,

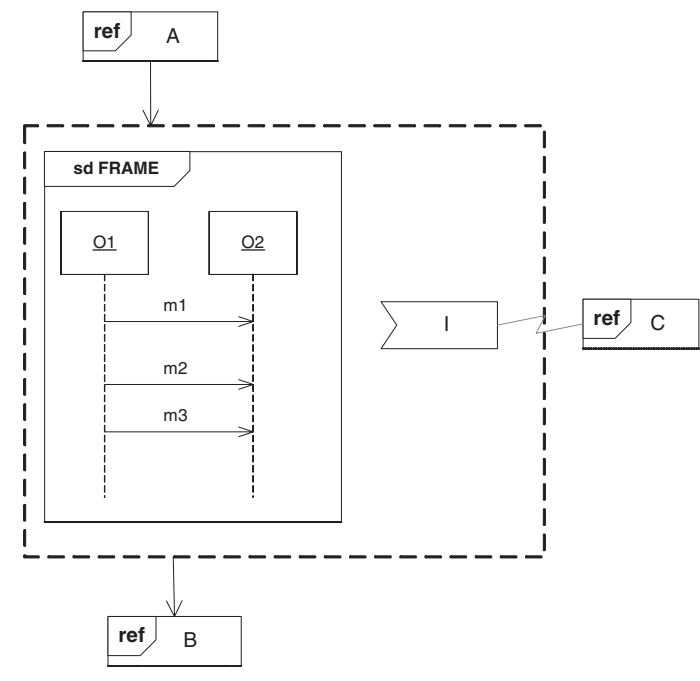

Figure 1. An example of interruptable region.

which is represented as an interface component named I, while object $\mathrm{O} 1$ and $\mathrm{O} 2$ are exchanging messages to each other. Then, the message exchange between those objects is stopped, and the component $\mathrm{C}$, which is connected to the component I, is executed.

\section{Creating Operational Architecture}

Figure 2 shows how our operational architecture is constructed. The inputs of our construction method are scenarios. For each use case, there are alternative scenarios as well as the main success scenario. So there are several scenarios for an use case. Our construction method begins with constructing the scenario-level operational architectures from each scenario. Then the use-case-level operational architecture for an use case are constructed by composing those scenario-level operational architectures. Finally, the usecase-level operational architectures are composed to make the system-level operational architecture. In this section, we describe how a scenario-level operational architecture for a scenario is described, and define composition functions for scenario-level operational architectures and for use-caselevel ones.

\subsection{Scenario-level Operational Architec- ture}

A scenario for embedded software describes how the system reacts for a sequence of input events from the environment $[3,16]$. It is a sequence of input events, system reactions, and output events. The input and output of events can be represented as interface components and the 


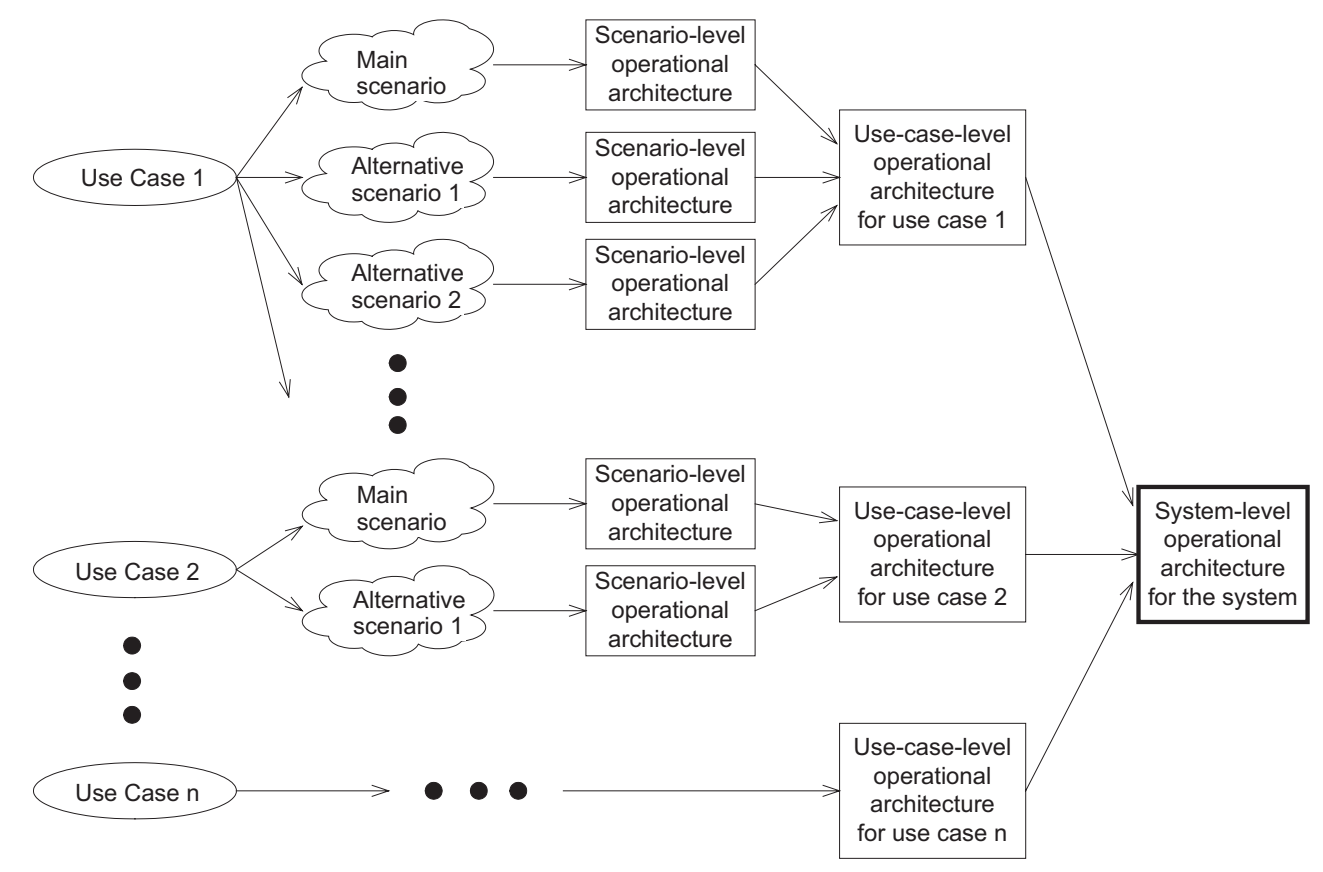

Figure 2. The overview of operational architecture construction.

system reactions as functional components in $\mathcal{C}$. So a scenario can be represented as a set of components and a set of ordinary transitions between them. As an example, consider following scenario:

1. An external system $\mathrm{S}$ sends event E1 to the system.

2. The system do some action A.

3. The system sends event E2 to S.

The scenario-level operational architecture for this scenario can be formalized as follows.

$$
\begin{aligned}
\mathcal{A}=(\mathcal{C}, & \left.\mathcal{R}, q_{\text {init }}, q_{\text {final }}, \mathcal{S}\right) \text { where } \\
\bullet & \mathcal{C}=\left\{\text { Receive }_{E 1}, \text { Action }_{A}, \text { Send }_{E 2}\right\} \\
\bullet & \mathcal{R}=\left\{\left(q_{\text {init }}, O, \text { Receive }_{E 1}, \text { null }\right)\right. \\
& \left(\text { Receive }_{E 1}, O, \text { Action }_{A}, \text { null }\right) \\
& \left(\text { Action }_{A}, O, \text { Send }_{E 2}, \text { null }\right) \\
& \left.\left(\text { Send }_{E 2}, O, q_{\text {final }}, \text { null }\right)\right\} \\
\bullet & \mathcal{S}=\varnothing
\end{aligned}
$$

Here, the components Receive R1 $_{1}$ and $S e n d_{E 2}$ are interface components and Action $_{A}$ is a functional component.

\subsection{Composing Scenario-level Operational Architecture}

There are several scenarios for an use case; the main success scenario and several alternative scenarios. Each one has its own scenario-level operational architecture. Those scenario-level operational architectures should be composed to describe use-case-level one for the use case. In our approach, we first compose the scenario-level operational architecture for the main success scenario and the one for an alternative scenario. Then, we compose it and another one. This binary composition continues until all the scenario-level operational architectures are composed.

When composing two scenario-level operational architectures, the relationship between them should be identified. It can be referred by examining corresponding scenarios. We identified two kinds of relationship between two scenarios; conditional and interruptable relations. The two scenarios have conditional relations when one scenario occurs when certain condition is not satisfied in another one. If one scenario occurs when the system is interrupted, which means that it receives an external event from the environment, while it performs another scenario, they have interruptable relations. We define first the composition function for conditional relationship. Assume that we have two scenario-level operational architectures $\mathcal{A}_{x}=$ $\left(\mathcal{C}_{x}, \mathcal{R}_{x}, q_{\text {init-x }}, q_{\text {final }-x}, \mathcal{S}_{x}\right)$ for the main success sce- 
nario and $\mathcal{A}_{y}$ for an alternative scenario that occurs when certain condition is not satisfied. If the decision making occurs after a component $c_{d} \in \mathcal{C}_{x}$, guard conditions for each branch are $T_{x}$ and $T_{y}$, and $c_{m} \in \mathcal{C}_{x}$ is a component that merges the two branches, then the composition function is defined as follows.

Definition 2 (Composition functions for conditional relationship).

Inputs

- $\mathcal{A}_{x}, \mathcal{A}_{y}$ : Scenario-level operational architectures to be composed

- $c_{d} \in \mathcal{C}_{x}:$ A component after which the decision making occurs

- $c_{m} \in \mathcal{C}_{x}:$ A component which merges the two branches

- $T_{x}, T_{y}$ : Guard conditions for branches in $\mathcal{A}_{x}$ and $\mathcal{A}_{y}$

Outputs

- $\mathcal{A}_{z}$ : The composed operational architecture

$$
\begin{aligned}
& \frac{\text { Algorithm }}{\mathcal{C}_{z} \leftarrow \mathcal{C}_{x} \cup \mathcal{A}_{y} ;} \\
& c_{e} \leftarrow \text { a component in } \mathcal{C}_{x} \text { such that } \\
& \left(c_{d}, O, c_{e}, \varnothing\right) \in \mathcal{R}_{x} ; \\
& \quad \mathcal{R}_{z} \leftarrow\left(\mathcal{R}_{x}-\left\{\left(c_{d}, O, c_{e}, \varnothing\right)\right\}\right) \cup\left\{\left(c_{d}, D, c_{e}, T_{x}\right)\right\} ; \\
& c_{n} \leftarrow \text { a component in } \mathcal{C}_{x} \text { such that } \\
& \left(c_{n}, O, c_{m}, \varnothing\right) \in \mathcal{R}_{x} ; \\
& \mathcal{R}_{z} \leftarrow\left(\mathcal{R}_{z}-\left\{\left(c_{n}, O, c_{m}, \varnothing\right)\right\}\right) \cup\left\{\left(c_{n}, M, c_{m}, \varnothing\right)\right\} ; \\
& \mathcal{R}_{z} \leftarrow \mathcal{R}_{z} \cup\left\{\left(c_{d}, D, \mathcal{A}_{y}, T_{y}\right),\left(\mathcal{A}_{y}, M, c_{m}, \varnothing\right)\right\} ; \\
& q_{\text {init-z }} \leftarrow q_{\text {init-x }} ; \\
& q_{\text {final-z }} \leftarrow q_{\text {final-x }} ; \\
& \mathcal{S}_{z} \leftarrow \mathcal{S}_{x} ; \\
& \mathcal{A}_{z} \leftarrow\left(\mathcal{C}_{z}, \mathcal{R}_{z}, q_{\text {init-z }}, q_{\text {final }-z}, \mathcal{S}_{z}\right) ;
\end{aligned}
$$

Figure 3 shows the graphical representations of two example scenario-level operational architectures and their composition result.

Now we define the composition function for interruptable relationship. Again, assume that we have two scenariolevel operational architectures $\mathcal{A}_{x}$ for the main success scenario and $\mathcal{A}_{y}$ for an alternative scenario that occurs when the system receives certain external events while the system executes certain component of $\mathcal{A}_{x}$. If the component that is in charge of receiving the event is $c_{i n t}$, components in $\mathcal{C}^{6} \subseteq \mathcal{C}_{x}$ can be interruptable by that event and $c_{m} \in \mathcal{C}_{x}$ is a component that merges the two branches, then the composition function is defined as follows.

Definition 3 (Composition functions for interrupts). Inputs

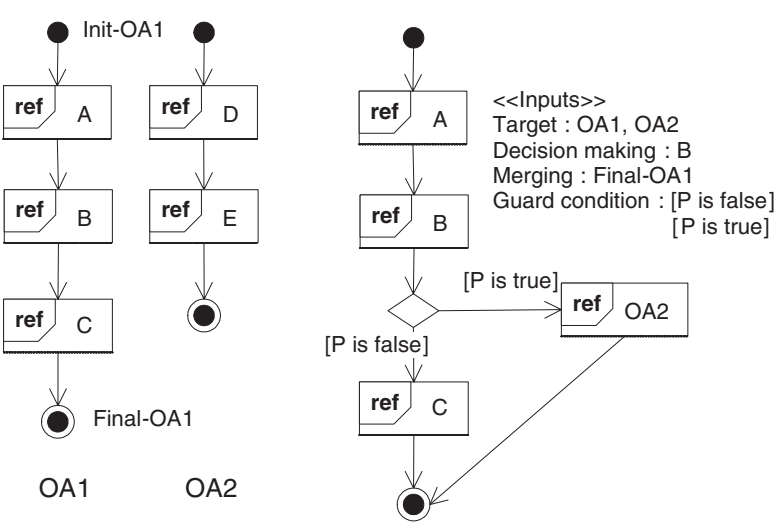

Figure 3. Two example scenario-level operational architectures and their composition.

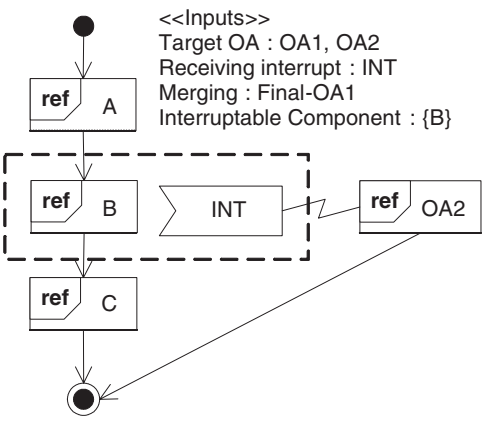

Figure 4. The composition result for an interrupt.

- $\mathcal{A}_{x}, \mathcal{A}_{y}$ : Scenario-level operational architectures to be composed

- $c_{\text {int }}$ : A component that receives external event

- $c_{m} \in \mathcal{C}_{x}:$ A component which merges the two branches

- $\mathcal{C}^{\prime} \subseteq \mathcal{C}_{x}$ : Set of interruptable components

Outputs

- $\mathcal{A}_{z}$ : The composed operational architecture

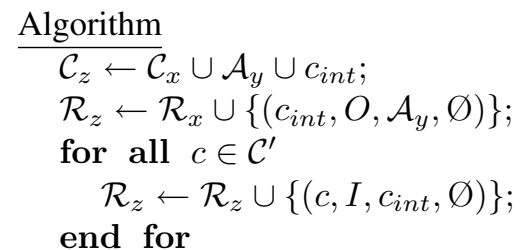




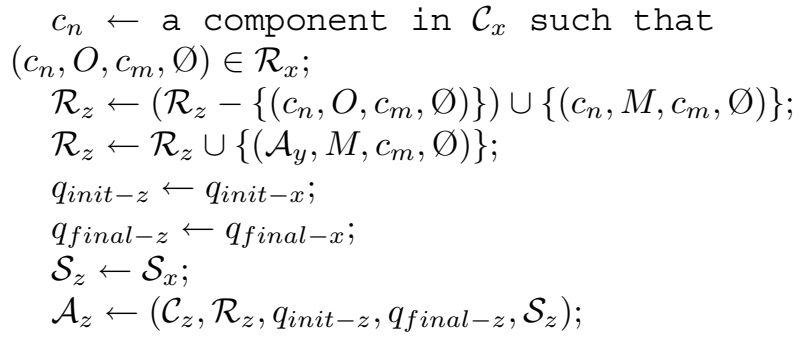

Figure 4 shows the composition result for interruptable relationship of the example scenario-level operational architectures in Figure 3.

With these two composition functions, we can compose scenario-level operational architectures for an use case, thus create an use-case-level operational architecture for the use case. What composition function to use for the composition of two scenario-level operational architectures can be decided by examining the relationship of corresponding scenarios.

\subsection{Composing Use-case-level Operational Architectures}

Now we have use-case-level operational architectures for use cases. They should be composed to describe the systemlevel operational architecture for the system. To do that, the relationships between them, which are equal to those between use cases, should be identified. Basically, use cases are independent to each other. Among them, some use cases have include or extend relationships. In our approach, the use-case-level operational architecture for an included use case is already included in that of the including use case, since the included use case is a part of the including use case. Also the use-case-level operational architecture for an extended use case is composed with that of the extending use case. This is because the scenarios for the extended use case is depicted as the form of an alternative scenario of the extending use case. So in this step, we do not consider the use-case-level operational architectures of included use cases and extended ones. Except the included and extended use cases, all use cases are independent to each other. Thus all the use-case-level operational architectures are composed in parallel. We define a function for the parallel composition of all use-case-level operational architectures.

Definition 4 (Composition functions for use cases). Inputs

- $\mathcal{A S}$ : Set of use-case-level operational architectures to be composed

Outputs

- $\mathcal{A}_{z}$ : The composed operational architecture

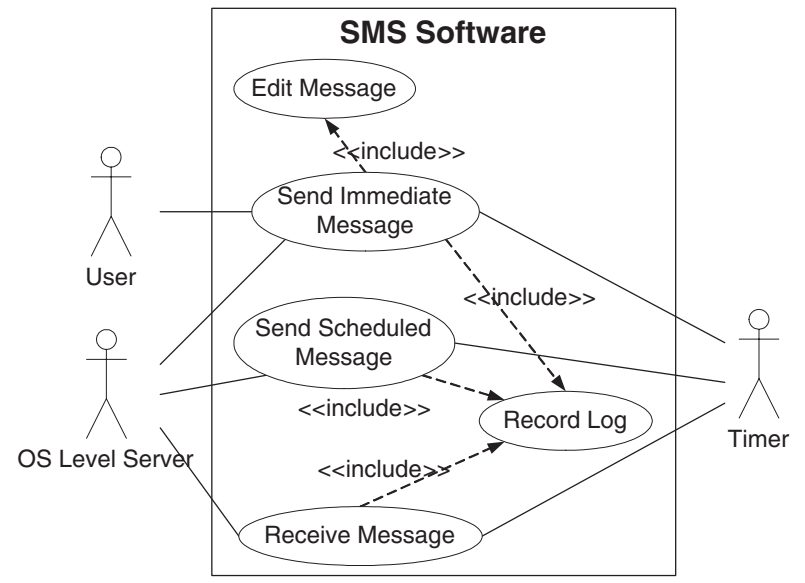

Figure 5. The use case diagram for SMS software.

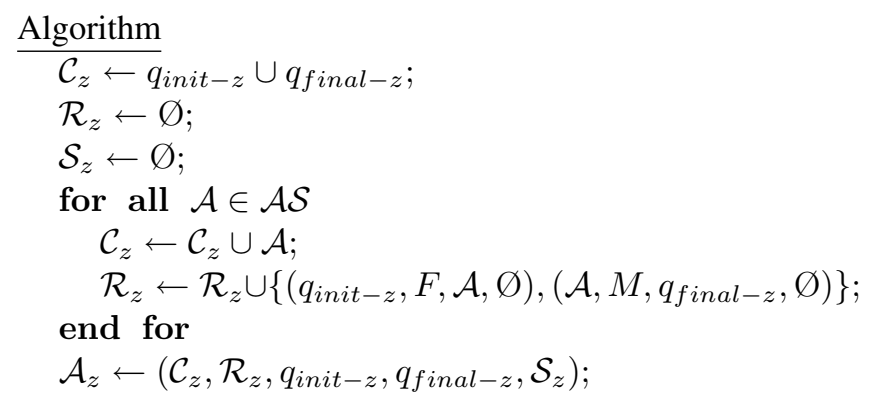

\subsection{Example}

We constructed an example operational architecture. The target is SMS(Short Message Service) software on mobile phone. As shown in Figure 5, we identified three actors and five use cases. For each use case, we described the main success scenario and alternative scenarios. The total number of scenarios is 17 .

For each scenario, we constructed the scenario-level operational architecture. Then, we composed them to create use-case-level operational architecture for each use case. Figure 6 shows the use-case-level operational architecture for 'Send Immediate Message' use case, which is represented using UML 2.0 interaction overview diagram. The functional components and reference components are depicted as InteractionUse node. If an interface component is in charge of receiving external event, it is depicted as $A c$ ceptEventAction node. If not, it is represented as SendSignalAction node. The scenario-level operational architecture for the main success scenario was composed with three scenario-level operational architectures; named as CancelMessage, AbortSending and HandleSendingFailure. 


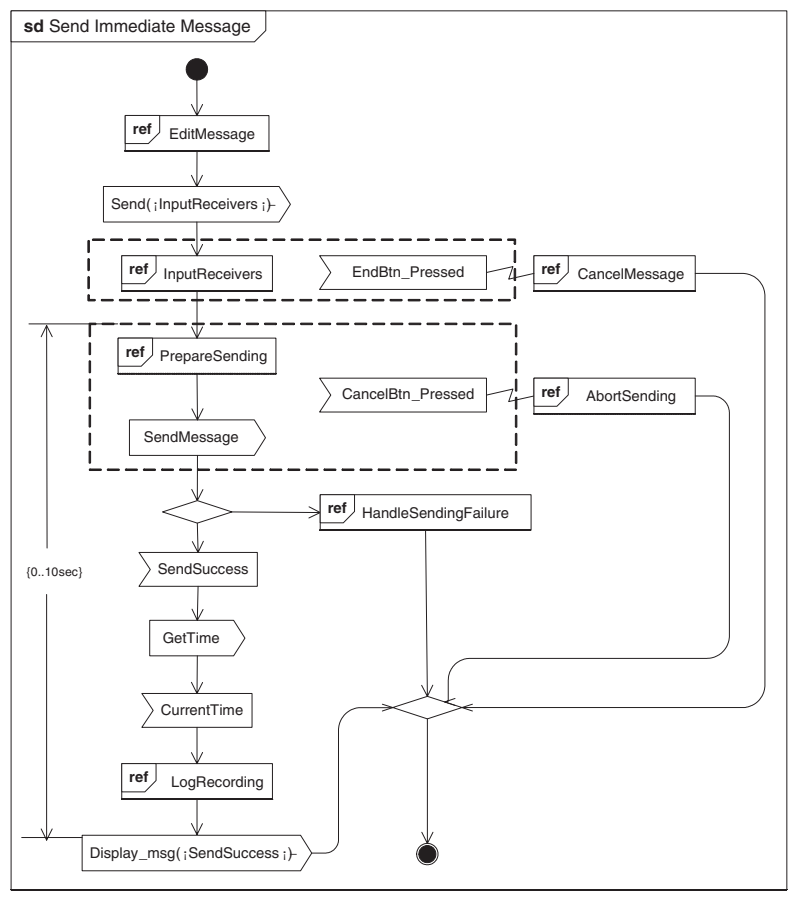

Figure 6. The operational architecture for 'Send Immediate Message' use case.

After creating use-case-level operational architecture for each use case, we composed all of them. Two use cases; 'Edit Message' and 'Record Log' use cases, have include relationships with other use cases. Their corresponding usecase-level operational architectures are not composed, since they are already included in other ones. Figure 7 shows the system-level operational architecture for the SMS software.

\section{Modeling based on Operational Architec- ture}

In this section, we describe modeling of embedded software based on operational architecture. We can derive structured models as well as object-oriented models from our operational architecture. Since the operational architecture focuses on interactions between functions, thus only the behavioral models can be derived. We describe how those models could be derived in detail.

\subsection{Structured Modeling}

In structured analysis and design, data flow models are used to describe the behavior of the system. We can derive data flow models from the operational architecture. The basic idea is that mapping functional components and refer-

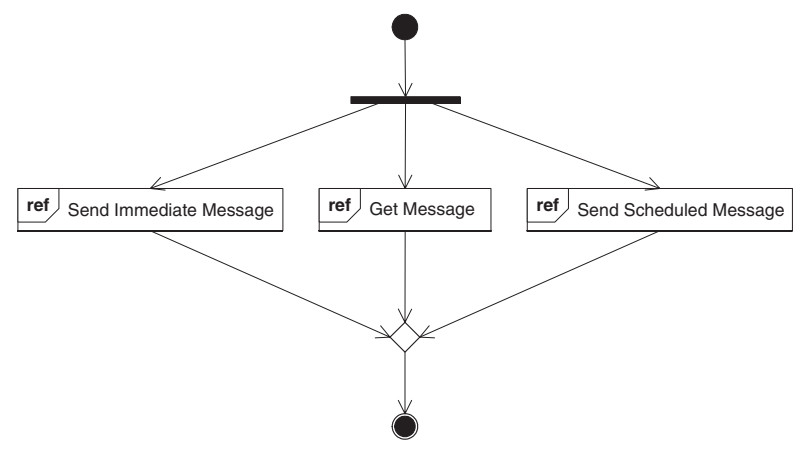

Figure 7. The operational architecture for SMS software.

ence components to transformations. Since the functional components represent some internal actions in the system, they could be mapped to transformations in data flow models. Also the reference components representing nested operational architectures could be mapped to transformations which can be decomposed.

Here we have two issues to consider. The first thing is how to map an interface component. An interface component is in charge of communicating with the environments. So it could be mapped to a data flow or an event flow. If it exchanges some values, such as current temperature, with the environments, it may be mapped to a data flow. If it sends/receives a simple event such as 'TurnOn' and 'TurnOff', it could be regarded as an event flow or a data flow with parameters. Another thing to consider is identifying data flows between use-case-level operational architectures representing independent use cases. The data dependencies between use cases are not identified in scenarios. So, through the detailed analysis, the modeler have to what data are exchanged between them. If none of them exist, the transformations representing independent use-caselevel operational architectures are modeled as independent ones.

For the clear explanation, we borrowed the requirements and use cases for cruise control system from [10]. In this system, there are two use case packages. We only considered 'CruiseControl' use case packages. In the package, as shown in Figure 8, there are four use cases. The system-level operational architecture for this use case package is composed of four reference components representing nested use-case-level operational architectures for use cases. Through the examination of the requirements, we identified several data flows among those use cases. The 'Determine Distance and Speed' use case receives inputs from the 'Update Shaft Rotation Count' and 'Perform Calibration' use cases and calculate the Cumulative Distance 


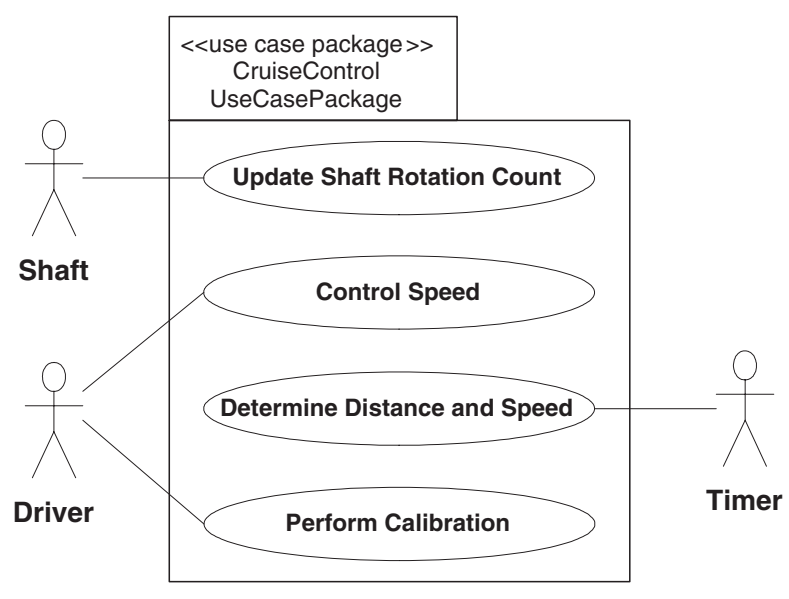

Figure 8. The use case model of cruise control use case package in [10].

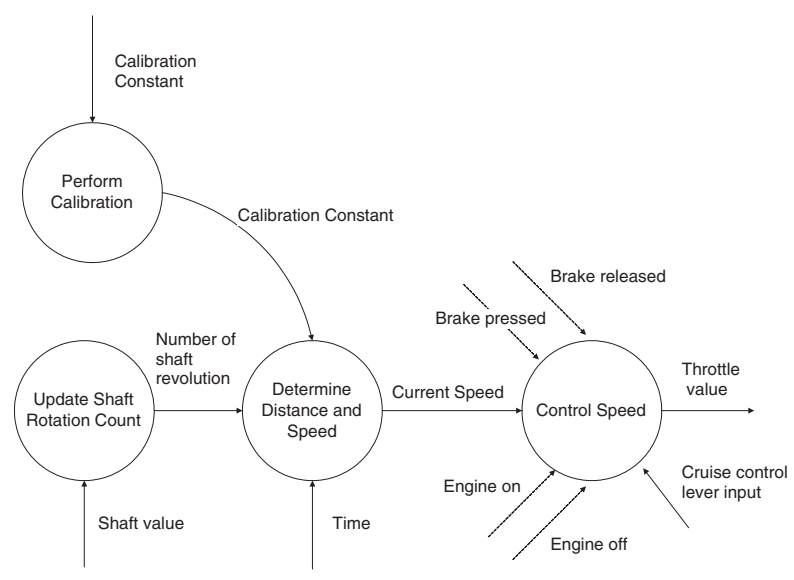

Figure 9. The derived data flow model.

traveled by the car and Current Speed of the car. And the Current Speed data is needed by the 'Control Speed' use case. With this information, we constructed initial data flow model, as shown in Figure 9. We also indicated data flows representing interface components in nested operational architectures. The external event from the cruise control lever is represented as a data flow with parameter, and the events from the brake and engine are modeled as event flows. Compared to the data flow models for the same system in [9], our derived model do not cover all data flows. Although it needs elaboration, this could be used as an initial sketch.

\subsection{Object-Oriented Modeling}

Deriving object-oriented behavior model from the operational architecture is straightforward. As we presented earlier, our operational architecture can be depicted using UML 2.0 interaction overview diagram. So the operational architecture itself can be regarded as high-level behavior model. The low-level behavior model can be constructed by realizing each functional component in the scenario-level operational architecture with UML 2.0 sequence diagrams. We have already suggested interaction-based behavior modeling approach with the interaction overview diagrams and the sequence diagrams[12].

\section{Related Works}

There exist approaches to model embedded software based on architecture. Frick et al.[8] presented architecture design for embedded control software with UML 2.0. Their focus is on modularization and variant design. The modules are represented by composite classes with ports. The services of the module are realized in terms of a statemachine and/or attributes and operations. Colbert et al.[4] proposed an architectural design language for specifying embedded software. With their language, MetaH, the user can model the hardware and software components of an application, the connections between them, and the execution characteristics of each component.

The role of our operational architecture is not different from that of above approaches, in the sense that it follows the definitions of software architecture by Kazman[1] It is high-level design and overall structure of the system. It defines software components, representing functions or activities, and their relationships. And, as we described earlier, it provide guidelines for the detailed design. The difference is that our operational architecture focuses on dynamic behavior, especially interactions with the environments, while others mainly focus on static structure of the system. Those interactions are derived directly from the requirements; i.e use cases, and both the structured models and object-oriented models can be derived smoothly from our operational architecture. This gives traceability between the requirements and the design model.

\section{Conclusion}

In this paper, we proposed an approach to model embedded software based on operational architecture, which focuses on the activities and the interaction between activities. We formally defined the operational architecture and described how it reflects scenarios. Also we define composition functions to merge operational architectures. Our 
operational architecture is constructed directly from the use case scenarios including alternative ones. It gives traceability between requirements and design model, thus the addition of new functionalities and the modification of existing ones can be done easily. And, by incorporating alternative scenarios, our operational architecture can reflect user requirements more effectively. Since our approach enables hierarchical modeling by nesting the operational architecture, the complexity of the model can be reduced.

We can produce both structured models and objectoriented model from the operational architecture. In structured design, each functional component can be mapped to a transformation in data flow model. So the operational architecture can mapped to data flow model with some elaboration. On the other hand, in object-oriented design, the operational architecture itself, which are graphically represented using UML 2.0 notations, can be regarded as highlevel behavior model. The detailed behavior model can be constructed by realizing each functional component in the operational architecture with UML 2.0 sequence diagrams.

Several works should be done to mature our approach. Firstly, we identified two kinds of relationships between two scenarios of an use case. It is possible that two scenarios have another relationships implicitly such as priority or resource contention between them. We will refine our approach to reflect those relationships. And we plan to apply our approach to the development of multi-processor system-on-chip(MPSoC) embedded software. To do that, we should investigate the method of partitioning and deploying the operational architecture according to the distributed hardware components.

\section{ACKNOWLEDGEMENT}

This work was supported in part by IT Leading R\&D Support Project funded by Ministry of Information and Communication, Republic of Korea and by the MIC (Ministry of Information and Communication), Korea, under the ITRC (Information Technology Research Center) support program supervised by the IITA(Institute of Information Technology Assessment).

\section{References}

[1] L. Bens, P. Clements, and R. Kazman. Software Architecture in Practice 2nd Edition. Addison-Wesley, 2003.

[2] M. Blaha and J. R. Rumbaugh. Object-Oriented Modeling and Design with UML. Prentice-Hall, 2004,

[3] K. S. Cheung, T. Y. Cheung, and K. O. Chow. A petri-net based synthesis methodology for use-case-driven system design. IEEE Transactions on Software Engineering, 79, 2006.

[4] E. Colbert, B. Lewis, and S. Vestal. Developing evolvable, embedded, time-critical systems with metah. 34th Interna- tional Conference on Technology of Object-Oriented Languages and Systems(TOOLS 34), August 2000.

[5] B. Douglass. Real-Time UML 3rd edition. Addison-Wesley, 2004.

[6] H. Eriksson, M. Penker, B. Lyons, and D. Fado. $U M L^{T M} 2$ Toolkit. Wiley, 2003.

[7] M. Fowler. UML Distilled 3rd Edition. Addison-Wesley, 2003.

[8] G. Frick, B. Scherrer, and D. Muller-Glaser. Designing the software architecture of an embedded system with uml 2.0. UML 2004 Workshop on Software Architecture Description and $U M L$, October 2003.

[9] H. Gomaa. Software Design Methods for Concurrent and Real-Time Systems. Addison-Wesley, 1996.

[10] H. Gomaa. Designing Concurrent, Distributed, and RealTime Application with UML. Addison-Wesley, 2000.

[11] B. Graaf, M. Lormans, and H. Toetenel. Embedded software engineering: The state of the practice. IEEE Software, November 2003.

[12] S. U. Jeon, J. E. Hong, and D. H. Bae. Interaction-based behavior modeling of embedded software using uml 2.0. Proceedings of the Ninth IEEE International Symposium on Object-Oriented Real-Time Distributed Computing, pages 351-358, 2006.

[13] E. A. Lee. Embedded software. Advances in Computers, 56, 2002.

[14] OMG. UML 2.0 Superstructure Specification (formal/0507-04). Available from : http://www.omg.org.

[15] T. Schattkowsky and W. Muller. Model-based design of embedded systems. Proceedings of the Seventh IEEE International Symposium on Object-Oriented Real-Time Distributed Computing, 2004.

[16] S. Uchitel, J. Kramer, and J. Magee. Synthesis of behavioral models from scenarios. IEEE Transactions on Software Engineering, 29(2), February 2003. 